histone acetyltransferases (anacardic acid) and deacetylases (trichostatin A). Binding patterns of monoclonal ACPAs, both whole and $F\left(a b^{\prime}\right) 2$ fragments were analyzed in synovial biopsies obtained from both healthy donors and RA patients.

Results: Three out of four tested individual ACPA were able to promote fibroblast migration. Five out of nine tested monoclonal ACPAs stimulated fibroblast migration. One of these antibodies, clone 1325:01B09 is characterized by cross-reactivity to citrullinated, homocitrullinated and acetylated targets. The effect of 1325:01B09 on fibroblast migration was completely abolished by $\mathrm{Cl}$-amidine or by pre-incubating the antibody with citrullinated fibrinogen or histone but not citrullinated enolase or vimentin. Despite the cross-reactivity to acetylated epitopes, neither anacardic acid nor trichostatin A could modulate the 1325:01B09 effect on fibroblast migration. $F(a b ') 2$ fragments of this antibody stimulated fibroblast migration and labelled podoplanin-positive fibroblasts in inflamed RA synovium similarly to the intact antibody, indicating an Fc-independent effect.

Conclusion: The effect on fibroblast mobility was likely to be mediated by binding to citrullinated epitopes but not through $\mathrm{Fc}_{\mathrm{c}}$ receptors. Detection of fibroblast modulating ACPAs in majority of RA patients indicated that fibroblasts might be key cellular targets in disease pathogenesis, although individual variability might exist in the composition of ACPA cellular targets.

References:

[1] Sun M, Rethi B, Krishnamurthy A, et al. Anticitrullinated protein antibodies facilitate migration of synovial tissue-derived fibroblasts. Ann Rheum Dis 2019;78(12):1621-31. doi: 10.1136/annrheumdis-2018-214967 [published Online First: 2019/09/05]

[2] Amara K, Lena Israelsson, Ragnhild Stålesen, et al. A Refined Protocol for Identifying Citrulline-specific Monoclonal Antibodies from Single Human B Cells from Rheumatoid Arthritis Patient Material. Bio-protocol 2019;9(16)

Disclosure of Interests: Meng Sun: None declared, Bence Réthi: None declared, Akilan Krishnamurthy: None declared, Vijay Joshua: None declared, Alexandra Circiumaru: None declared, Marianne Engström: None declared, Caroline Grönwall: None declared, Vivianne Malmström Grant/research support from: VM has had research grants from Janssen Pharmaceutica, Khaled Amara: None declared, Lars Klareskog: None declared, Heidi Wähämaa: None declared, Anca Catrina: None declared

DOI: 10.1136/annrheumdis-2020-eular.5343

\section{FRI0006 \\ ASSESSING PRO-INFLAMMATORY PROPERTIES OF H-FERRITIN BY EX VIVO AND IN VITRO OBSERVATIONS}

P. Ruscitti ${ }^{1}$, P. Di Benedetto ${ }^{1}$, O. Berardicurti ${ }^{1}$, N. Panzera ${ }^{1}$, F. Sensini ${ }^{1}$, P. Cipriani ${ }^{1}$, R. Giacomelli ${ }^{1}$. 'University of L'Aquila, Department of Biotechnological and Applied Clinical Sciences, L'Aquila, Italy

Background: The concept of 'hyperferritinemic syndrome' has recently been proposed, suggesting high levels of ferritin as pathogenic pro-inflammatory mediator [1] Ferritin is an intracellular iron storage protein, comprising 24 subunits, heavy $(\mathrm{H})$ and light $(\mathrm{L})$ based on molecular weight. The $\mathrm{H}$-/L subunits ratio may be different in tissues, since the ferritin enriched in $L$ subunits (L-ferritin) and the ferritin enriched in $\mathrm{H}$ subunits ( $\mathrm{H}$-ferritin) may be observed in different tissues, according to pathophysiologic status [1].

Objectives: We aimed to assess the presence of $\mathrm{H}$ - and L-ferritin as well as of CD68/H-ferritin and CD68/L-ferritin in bone marrow (BM) biopsies of adult macrophage activation syndrome (MAS) patients. In the same patients, we matched the findings of BM biopsies with sera to identify the main represented subunits of ferritin. Furthermore, we evaluated effects of ferritin, L-ferritin, and $\mathrm{H}$-ferritin on human monocytes, assessing pro- and anti-inflammatory cytokines, and expression of NLRP3 inflammasome. Finally, we checked the ability of monocytes, which were treated with ferritin, to stimulate or not the proliferation of peripheral blood mononuclear cells (PBMCs).

Methods: Immunofluorescence analysis was performed to investigate the tissue presence of $\mathrm{L}$ - and $\mathrm{H}$-ferritin in $\mathrm{BM}$ biopsies as well as of $\mathrm{CD} 68 / \mathrm{H}$-ferritin and CD68/L-ferritin. Liquid chromatography mass spectrometry (LC-MS/ MS) based proteomics was performed to identify $\mathrm{L}$ - and $\mathrm{H}$-ferritin in sera proteins. Human monocytes were cultured with M-CSF for 7 days and, after that, treated with ferritin, $\mathrm{H}$-ferritin, and L-ferritin at $10 \mathrm{nM}$, for 120 and 240 minutes. After stimulation, IL-1 $\beta$, IL-6, IL-10, IL-12, IFN- $\gamma$, TGF- $\beta$, TNF, and VEGF were assessed by RT-PCR and, in case of positive finding, evaluated by western blot. NLRP3 inflammasome was also assessed. Finally, the proliferation of PBMCs when co-cultured with ferritin-treated monocytes was tested by a specific proliferation assay.
Results: Immunofluorescence showed an increased $\mathrm{H}$-ferritin expression in the BMs of MAS patients, whereas L-ferritin did not. Conversely, LC-MS/MS identified that the L-ferritin was the dominant form, after stringent probability matching. In vitro, $\mathrm{H}$-Ferritin induced a significant increased expression of IL-1 $\beta$, IL-6, $\mathrm{IL}-12$, and TNF after 240 minutes. Ferritin also induced a significant increased expression of IL-1 $1 \beta$, IL-6, IL-12, and TNF after 240 minutes. Effects on pro-inflammatory cytokines were more marked with $\mathrm{H}$-ferritin than ferritin. Conversely, no significant effects were retrieved analysing IFN- $\gamma$, IL-10, TGF- $\beta$, and VEGF after 240 minutes, after ferritin and $\mathrm{H}$-ferritin stimulation. Furthermore, both ferritin and $\mathrm{H}$-ferritin induced a direct effect on NLRP3 inflammasome. Finally, monocytes, which were treated with $\mathrm{H}$-ferritin, stimulated the proliferation of co-cultured PBMCs.

Conclusion: In our work, results showed the presence of $\mathrm{H}$-ferritin and CD68/H-ferritin cells in BM biopsies of MAS patients, by immunofluorescence. Conversely, LC-MS/MS identified L-ferritin in sera proteins of those patients. Furthermore, pro-inflammatory effects of ferritin and, particularly, of $\mathrm{H}$-ferritin on human monocytes were observed in vitro, increasing pro-inflammatory cytokines and NLRP3 inflammosome. Finally, $\mathrm{H}$-ferritin-treated monocytes stimulated the proliferation of co-cultured PBMCs.

References:

[1] Rosario C, et al. BMC Med 2013; 11:185.

Disclosure of Interests: Piero Ruscitti Grant/research support from: Pfizer, Speakers bureau: BMS, MSD, Ely Lilly, SOBI, Paola Di Benedetto Grant/research support from: Paola Di Benedetto received grant from Dompè outside this work. Onorina Berardicurti: None declared, Noemi Panzera: None declared, Federica Sensini: None declared, Paola Cipriani Grant/research support from: Actelion, Pfizer, Speakers bureau: Actelion, Pfizer, Roberto Giacomelli Grant/research support from: Actelion, Pfizer, Speakers bureau: Abbvie, Roche, Actelion, BMS MSD, Ely Lilly, SOBI, Pfizer DOI: 10.1136/annrheumdis-2020-eular.3473

\section{FRI0007 \\ MOSPD2: A NOVEL THERAPEUTIC TARGET FOR THE TREATMENT OF RHEUMATOID ARTHRITIS}

N. Yacov ${ }^{1}$, Y. Salem ${ }^{1}$, O. Prophate-Meira ${ }^{1}$, P. Kafri ${ }^{1}$, E. Breitbart ${ }^{1}$, I. Mendel ${ }^{1}$ ${ }^{1}$ VBL Therapeutics, Modi'In, Israel

Background: Rheumatoid arthritis (RA) is a chronic inflammatory autoimmune disease with an unknown etiology that affects the joints. B and T lymphocytes are major drivers of arthritis, mediated through their secretion of antibodies against self-antigens and the production of pro-inflammatory cytokines. Nevertheless, innate immune cells play an important role in disease progression, and recruitment of blood monocytes/macrophages was highly correlated with tissue damage in RA patients ${ }^{1,2}$. Once infiltrated into the joint, monocytes can produce proinflammatory cytokines and chemokines that further escalate the inflammatory process and tissue damage. Consistent with that, genetic and pharmacological intervention targeting CCR2 and CCR5, chemokine receptors which are abundantly expressed on monocytes, was shown to reduce disease severity in multiple preclinical models of $\mathrm{RA}^{3}$. Blocking monocyte migration in RA can be therefore an attractive therapeutic approach, if implied in a manner that can overcome the redundancy of chemokine receptors and ligands.

Motile sperm domain-containing protein 2 (MOSPD2) is a 518 amino acids single-pass membrane protein to which no function was ascribed up unti recently. Assessment of MOSPD2 in immune cell subsets showed it is expressed by monocytes and neutrophils. We previously discovered that MOSPD2 regulates human monocyte and neutrophil migration in a chemok ine-agnostic manner ${ }^{4}$.

Objectives: Using anti MOSPD2 mAbs, the role of MOSPD2 in mouse monocyte migration in-vitro and RA progression in mice was assessed.

Methods: In-vitro, MCP-1 and SDF-1 chemokines were used to induce migration of mouse splenic monocytes through a trans-well plate in the presence of anti MOSPD2 mAbs. In-vivo, DBA-1 mice were injected with type II collagen on days 0 and 21 to induce arthritis. Mice were then treated twice a week with anti MOSPD2 mAb, anti TNF-a or an isotype control antibody until study end (day 42). Animals were monitored for arthritis score, and upon sacrifice, hind limb joints were examined for pathology.

Results: Anti MOSPD2 mAbs significantly inhibited migration of mouse monocytes in-vitro. Treatment with anti MOSPD2 mAb after disease was already initiated, limited disease progression and significantly ameliorated clinical manifestation at the climax phase of the disease by more than $50 \%$ compared with isotype control and anti TNF-a treatment. Pathological examination revealed that treatment with anti MOSPD2 mAb reduced pannus formation and joint inflammation. 